\title{
Antibiotics in microbial coculture
}

\author{
Kenji Ueda and Teruhiko Beppu
}

Today, the frequency of discovery of new antibiotics in microbial culture is significantly decreasing. The evidence from whole-genome surveys suggests that many genes involved in the synthesis of unknown metabolites do exist but are not expressed under conventional cultivation conditions. Therefore, it is urgently necessary to study the conditions that make otherwise silent genes active in microbes. Here we overview the knowledge on the antibiotic production promoted by cocultivation of multiple microbial strains. Accumulating evidence indicates that cocultivation can be an effective way to stimulate the production of substances that are not formed during pure cultivation. Characterization of the promotive factors produced by stimulator strains is expected to give clues to the development of effective cultivation conditions for drug discovery.

The Journal of Antibiotics (2017) 70, 361-365; doi:10.1038/ja.2016.127; published online 19 October 2016

\section{INTRODUCTION}

Ever since the discovery of penicillin, antibiotics and many other kinds of biologically active substances have been obtained from microbial cultures. The divergent actions of these compounds have contributed to the current knowledge of the basic mechanisms of molecular interaction and therapeutic methods as well. This special issue contains studies dealing with the milestone works that have advanced the development of basic biological sciences and medicinal applications.

In contrast to the great successes in mining of useful natural products in the last century, the frequency of isolation of new compounds from microbial cultures is substantially decreasing in this century. ${ }^{1}$ In accordance with this situation, the pharmaceutical industry is changing the direction of drug development and reducing the weight of natural products among the resources for screening.

The factors limiting the chances of new discovery can be attributed to humans, not to microorganisms. Studies on the accumulating genomic information indicate that a large number of biosynthetic gene clusters are not expressed under the conventional culture conditions. ${ }^{1,2}$ Namely, the potential diversity of microbial products is still large, but scientists do not yet know how to fully take advantage of the abilities of microbes. This problem is due in part to the fact that the methods of modern microbiology still depend on the manipulation techniques developed by Robert Koch and colleagues, which relies on the single-colony isolation from solid agar plates and subsequent pure cultivation under laboratory conditions. We can imagine that the dependence on those artificial conditions restricts access to a number of microorganisms and/or their abilities.

To address the issue, attempts have been made to discover new substances specifically produced in coculture. In natural environments, microbes are living in diverse associations with other organisms. The modes of interaction include antagonism, commensalism and mutualism. Functions specifically correlating with such interactions may not get activated during conventional pure cultivation. Here we overview the current knowledge of antibiotic production specifically observed in microbial coculture and the possible mechanisms underlying this phenomenon.

\section{EFFECTIVENESS OF COCULTURE}

One of the studies on the effect of cocultivation on antibiotic production was carried out by Sonnenbichler et al., ${ }^{3}$ who researched the interactions between fungal strains. On the basis of the observation that the growth of two Basidiomycetes, Heterobasidion annosum and Gloeophyllum abietinum, proceeds in an antagonistic manner, the antimicrobial substances specifically produced in the coculture of the two fungal organisms were investigated. The result showed that the concentrations of oosponol and oospoglycol, the metabolites produced by $G$. abietinum, were remarkably elevated in coculture. ${ }^{3}$ Detailed characterization of the pure culture of each fungus revealed that the presence of oosponol or oospoglycol induced the synthesis of fomannosin in $H$. annosum. This effect represents the interactive mode in which the production of a secondary metabolite in one organism takes place after sensing of a secondary metabolite produced by another organism.

Burgess et al. ${ }^{4}$ studied the antagonistic response of marine bacteria to the challenge with terrestrial bacteria. Epibiotic bacterial strains isolated from the surface of seaweed were tested for their ability to produce antibiotics in response to the presence of living cells of pathogenic bacteria such as Staphylococcus aureus or Pseudomonas aeruginosa. The researchers expected that the ability of marine epibiotic bacteria to produce diverse antimicrobial substances may be stimulated by an antagonistic interaction with the cells of pathogenic bacteria, and that such substances may be candidates for new drugs against infectious diseases. In fact, those investigators found that a high proportion of marine bacterial isolates produces a higher amount of antibiotics if they are cultured in the presence of living cells 
of pathogenic bacteria. The evidence implied that the cocultivation is a promising strategy for drug mining.

We studied the interaction between strains of Streptomyces: Gram-positive filamentous soil bacteria. ${ }^{5}$ Streptomyces and related bacteria are characterized by the ability to produce a wide variety of secondary metabolites and to undergo complex cell differentiation resembling that of filamentous fungi. It is known that antibiotic production in some Streptomyces is triggered by a $\gamma$-butyrolactone autoinducer. The best-characterized inducer is A-factor (autoregulatory factor; 2-isocapryloyl-3 R-hydroxymethyl- $\gamma$-butyrolactone) produced by Streptomyces griseus. A-factor induces both the production of secondary metabolites and the formation of an aerial mycelium via its binding to the specific receptor ArpA. The binding of A-factor to ArpA results in the expression of a global transcriptional regulator called AdpA, which activates the transcription of genes encoding pathway-specific regulators. ${ }^{6}$

We studied the interaction between different Streptomyces strains expecting that small-molecule signaling, including that mediated by $\gamma$-butyrolactone inducers, would take place not only in an intraspecific but also interspecific manner within this group of complex bacteria capable of producing diverse chemical compounds. Our cross-stimulation assay between different strains of Streptomyces (Figure 1) demonstrated that the interspecific stimulation of antibiotic production and/or cell differentiation occurs at a high frequency. ${ }^{5}$ The stimulation appeared to be based on mutualistic rather than antagonistic interactions. One possibility is that a chemical factor produced
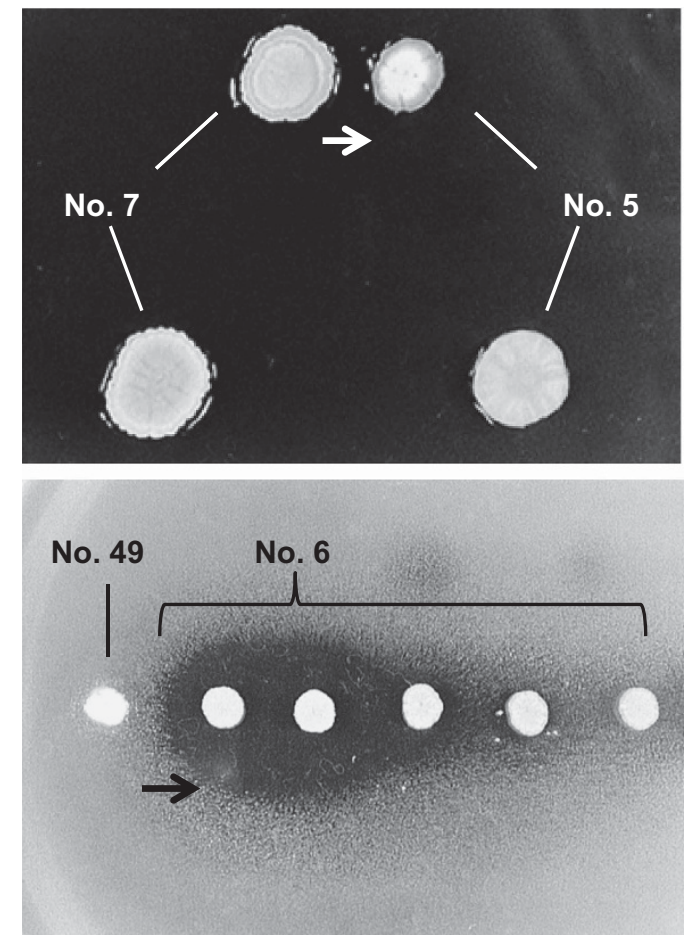

Figure 1 Cross-stimulation assays between two Streptomyces strains. Upper panel, an example of positive response in the paring analysis. Aerial mycelium formation of strain No 5 (responder) is stimulated when it was grown in close proximity to strain No 7 (stimulator). Lower panel, an example of positive response in the cross-feeding assay. Antibiotic production in strain No. 6 (receiver) is stimulated by the growth of strain no. 49 (stimulator). Antibiotic activity is visualized by the growth inhibition of Bacillus subtilis. For details see the original article. ${ }^{5}$. A full color version of this figure is available at The Journal of Antibiotics journal online. by the stimulator strain induces developmental events in the receiver strain. Our isolation study successfully identified two such stimulatory molecules (desferrioxamines and promomycin; see below).

Another analysis of the interactions involving Streptomyces strains was performed by Onaka et al. ${ }^{7}$, who discovered that cocultivation with mycolic-acid-containing bacteria represented by Tsukamurella causes marked stimulation of antibiotic production in various Streptomyces strains. Although the mechanism of stimulation has not yet been clarified, the attempts at cocultivating Streptomyces spp. with Tsukamurella led to the discovery of new substances (see below), indicating that this system offers promising conditions for drug mining.

\section{ANTIBIOTICS ISOLATED FROM MICROBIAL COCULTURE}

Table 1 summarizes antibiotics isolated from microbial cocultures. The early studies were focused on substances whose production is triggered by the antagonistic interaction of marine microorganisms. Istamycin production ${ }^{8}$ in Streptomyces tenjimariensis, a marine actinomycete, is enhanced by cocultivation with various marine bacteria. Pestalone ${ }^{9}$ and libertellenones ${ }^{10}$ were identified in the coculture of a marine-source fungus with a bacterial strain. Emericellamides ${ }^{11}$ were identified during the interaction between marine-source fungus and actinomycetes. Citrifelins ${ }^{12}$ were discovered in the coculture of two marine-source fungal isolates.

Fungal interactions were also applied to the strains of terrestrial origin. Acremostatins ${ }^{13}$ and secopenicillide $\mathrm{C}^{14}$ were discovered in a coculture of two fungi. Glionitrin $\mathrm{A}^{15}$ was discovered in the coculture of a fungal strain with a bacterial strain. $N$-formyl alkaloids ${ }^{16}$ and tetramic acid analogs ${ }^{17}$ were identified in the interaction of a fungus with actinomycetes. Recently, Hoshino et al. ${ }^{18,19,20}$ identified three novel classes of compounds-arcyriaflavin E, ${ }^{18}$ niizalactams ${ }^{19}$ and chojalactones ${ }^{20}$-in the coculture of Streptomyces spp. with Tsukamurella pulmonis.

The two indole derivatives with antibacterial activity, 1,1-bis (3'-indolyl)ethane and 2-bis( $3^{\prime}$-indolyl)indoxyl (Figure 2), were identified in our study on the coculture of two free-living bacteria, Geobacillus stearothermophilus and Symbiobacterium thermophilum. ${ }^{21}$ The latter is a syntrophic bacterium whose growth depends on the coculture with G. stearothermophilus. Originally, the coculture of the two bacterial organisms was obtained from a compost sample because of the presence of a thermostable tryptophanase. ${ }^{22}$ The tryptophanase producer could not be isolated by the conventional colony isolation method even though a distinctive tryptophan-degrading enzymatic activity existed in the original liquid culture. A detailed study of this phenomenon finally revealed that the tryptophanase producer was $S$. thermophilum, and that $S$. thermophilum was able to grow only under the coculture with G. stearothermophilus. Knowledge of the physiological, taxonomical and ecological properties of this unique syntrophic bacterium is reviewed in our recent article. ${ }^{23}$ Currently, we are capable of purely cultivating $S$. thermophilum by introducing a $\mathrm{CO}_{2}$-containing anaerobic atmosphere.

Although details are not yet known, 1,1-bis( $3^{\prime}$-indolyl)ethane and 2-bis ( $3^{\prime}$-indolyl)indoxyl are believed to be formed by the condensation of indole. The latter is generated by the tryptophanase-mediated process of tryptophan degradation. 1,1-Bis( $3^{\prime}$-indolyl)ethane and 2-bis $\left(3^{\prime}\right.$-indolyl)indoxyl are present at $6-7 \mu \mathrm{g} \mathrm{ml}^{-1}$ in the coculture of $S$. thermophilum and G. stearothermophilus, and at $0.4-0.9 \mu \mathrm{g} \mathrm{ml}^{-1}$ in the monoculture of $S$. thermophilum. The concentration of 1,1-bis ( 3 '-indolyl)ethane in the coculture is higher than that completely inhibiting pure growth of $S$. thermophilum. ${ }^{21}$ Thus, it appears likely to be that not only the formation of but also resistance to the 
Table 1 Antibiotics discovered in microbial coculture

\begin{tabular}{|c|c|c|c|}
\hline Product & Producer & Partner & Reference \\
\hline Istamycins A-B & S. tenjimariensis & Marine bacteria & Slatttery et al..$^{8}$ \\
\hline Pestalone & Pestalotia sp. & Antibiotic-resistant marine bacterium & Cueto et al..$^{9}$ \\
\hline Acremostatins $\mathrm{A}-\mathrm{C}$ & Acremonium sp. & Mycogone rosea & Degenkolb et al. ${ }^{13}$ \\
\hline Libertellenones A-D & Libertella sp & Marine $\alpha$-Proteobacterium & Oh et al. ${ }^{10}$ \\
\hline Indole derivatives & S. thermophilum & G. stearothermophilus & Watsuji et al. ${ }^{21}$ \\
\hline Emericellamides A-B & Emericella sp. & Salinispora arenicola & Oh et al. ${ }^{11}$ \\
\hline Glionitrin A & Aspergillus fumigatus & Sphingomonas sp. & Park et al. ${ }^{15}$ \\
\hline$N$-formyl alkaloids & A. fumigatus & Streptomyces peucetius & Zuck et al. ${ }^{16}$ \\
\hline Secopenicillide C & Penicillium pinophilum & Trichoderma harzianum & Nonaka et al. ${ }^{14}$ \\
\hline Tetramic acid analogs & Fusarium pallidoroseum & Saccharopolyspora erythraea & Whitt et al. ${ }^{17}$ \\
\hline Citrifelins A-B & Penicillium citrinum & Beauveria feline & Meng et al. 12 \\
\hline Arcyriaflavin E & Streptomyces cinnamoneus & Tsukamurella pulmonis & Hoshino et al. ${ }^{18}$ \\
\hline Niizalactams A-C & Streptomyces sp. & T. pulmonis & Hoshino et al. ${ }^{19}$ \\
\hline Chojalactones $\mathrm{A}-\mathrm{C}$ & Streptomyces sp. & T. pulmonis & Hoshino et al. ${ }^{20}$ \\
\hline
\end{tabular}<smiles>CC(c1c[nH]c2ccccc12)c1c[nH]c2ccccc12</smiles><smiles>O=C1c2ccccc2NC1(c1c[nH]c2ccccc12)c1c[nH]c2ccccc12</smiles>

Figure 2 Chemical structure of 1,1-bis(3'-indolyl)ethane (BIE) and 2-bis(3'-indolyl)indoxyl (BII).

antibacterial indole derivative depends on the cocultivation of $S$. thermophilum with G. stearothermophilus.

\section{COMPOUNDS STIMULATING ANTIBIOTIC PRODUCTION}

The aforementioned observation regarding the stimulation of antibiotic production because of Streptomyces interactions prompted us to isolate the promotive factors produced by the stimulator strains. To date, we have successfully identified two types of substances: desferrioxamines (siderophores) and promomycin (an ionophore). In addition, we recently found that ATP synthesis inhibitors have a potential to activate antibiotic production.

\section{Desferrioxamines}

In the course of aforementioned screening for a Streptomyces interaction that stimulates antibiotic production, we found that the vegetative and developmental growth of a strain of Streptomyces tanashiensis is remarkably promoted when it is grown in close proximity to $S$. griseus. It appears likely to be that a diffusible substance produced by $S$. griseus compensated for a certain deficiency in S. tanashiensis. The promotive factor was successfully isolated from the pure liquid culture of $S$. griseus and was identified as desferrioxamine E. ${ }^{24}$ Desferrioxamines are a group of siderophores often produced by Streptomyces. In a natural environment, the availability of iron to cells is quite limited, because of its insolubility. Hence, many organisms synthesize and secrete siderophores to capture and uptake ferric ions into the cell.

Presumably, the strain of $S$. tanashiensis lacks the ability to produce a siderophore but is capable of incorporating a ferrioxamine (a ferric-ion-bound form of a desferrioxamine) and delivering the ferric ion to ferric-ion-dependent processes. Furthermore, we performed screening for bacterial isolates with a desferrioxaminedependent phenotype and observed a stricter dependence of growth on the supply of desferrioxamine with regard to the isolates affiliated with Microbacterium. ${ }^{25}$ In an isolate of Janthinobacterium lividum, treatment with desferrioxamine does not strongly affect the growth but stimulates the production of violacein, a purple pigment antibiotic. In contrast to the positive effect, growth of some bacteria is significantly inhibited due to the presence of desferrioxamine. ${ }^{25}$ This finding suggests that those bacteria do not retain the ability to use ferrioxamine but have a different ferric-ion uptake mechanism.

Streptomyces interactions involving the synthesis of desferrioxamines have been extensively studied by Traxler et al. ${ }^{26}$, who characterized the chemical responses by using the nanospray desorption electrospray ionization and matrix-assisted laser desorption ionization-time-offlight imaging mass spectrometer. The analysis on model interaction between the colonies of Streptomyces coelicolor A3(2) and several actinomycetes strains demonstrated that the majority of secreted compounds associated with $S$. coelicolor colonies were unique, supporting the notion that interactive growth is an effective condition for the production of diverged secondary metabolites. Spectral networking based on the analytical results successfully deduced a family of unknown compounds produced by $S$. coelicolor. These compounds included at least 12 different desferrioxamines with acyl side chains of various lengths. The evidence indicates that the production of the novel desferrioxamines in $S$. coelicolor is triggered by the ferric depravation due to the production of a different type of siderophore by neighboring strains. ${ }^{26}$

\section{Promomycin}

Another compound discovered in a Streptomyces crosstalk assay is an ionophoric substance termed promomycin. ${ }^{27}$ Promomycin is produced by a strain closely related to Streptomyces scabrisporus, which serves as a stimulator strain for a strain related to Streptomyces griseorubiginosus. ${ }^{27}$ Structural analysis by the isolated activity principle 
revealed that the substance responsible for the stimulation (that is, promomycin) is a polyether related to lonomycin. Promomycin shows an antibiotic activity by itself; however, at subinhibitory concentrations, it stimulates antibiotic production in S. griseorubiginosus and several other Streptomyces strains. Thus, the activity of promomycin became an example of a new effect of an antibiotic at a subinhibitory concentration. The possibility of such effects was previously suggested by Davies et al. ${ }^{28}$

Similar promotion of antibiotic production was observed in relation to the dose of monensin. Monensin (Figure 3 ) is an ionophore widely used in animal husbandry as an agent promoting the growth of livestock and controlling chicken coccidiosis. Incubation with monensin at its subinhibitory concentration induces antibiotic production in various Streptomyces strains. ${ }^{29}$ Of these, we successfully isolated an antibiotic produced by $S$. griseorubiginosus and identified it as an isonitrile antibiotic previously reported in a patient (Figure 3). ${ }^{29}$ Although the details of the induction mechanism are not yet known, we expect that the treatment with monensin and related ionophores in such settings will stimulate the production of substances that are not produced under the conventional culture conditions.

\section{ATP synthesis inhibitors}

Recently, we reported that treatment with ATP synthesis inhibitors at a subinhibitory concentration induces actinorhodin production in respiratory mutants of $S$. coelicolor $\mathrm{A} 3(2) .{ }^{30}$ The role of the intracellular ATP level as a signal modulating Streptomyces physiology was originally proposed by Suh and colleagues. ${ }^{31} \mathrm{We}$ found that S. coelicolor mutants of cytochrome oxidase have a bald phenotype (defective in the development of an aerial mycelium and spore, and in production of secondary metabolites), and that the mutants contain high concentrations of intracellular ATP. Treatment with exogenous CCCP (carbonylcyanide $m$-chlorophenylhydrazone), a proton-uncoupling agent that inhibits ATP synthesis, restores the wild-type phenotype in the mutants. This evidence supports the viewpoint that a high intracellular ATP concentration serves as a negative signal for transition from the vegetative to developmental phase.

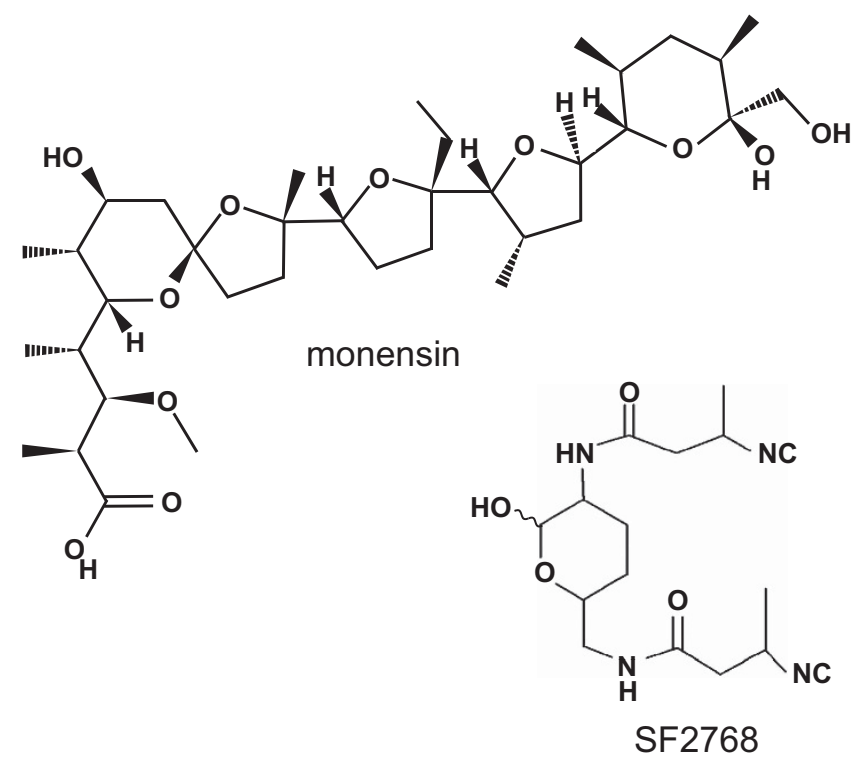

Figure 3 Chemical structure of monensin and SF2768, an isonitrile antibiotic whose production is stimulated by monensin.
Although it is not yet known how the ATP level is sensed, how the signal is transmitted to the genetic program controlling the onset of development and why the respiratory mutants accumulate a high level of ATP, it is likely to be that some signal from primary metabolism has a connection to the developmental regulatory network. Our finding of the inhibitory effect of an exogenous supply of ATP on the developmental growth of various Streptomyces spp. ${ }^{30}$ suggests that ATP signaling generally takes place. This observation in turn implies that modulation of the intracellular ATP level by means of inhibitors at an appropriate concentration may open the (usually closed) signaling gate and cause silent cells to initiate formation of secondary metabolites. This approach may become a cornerstone of a new methodology for drug mining.

\section{CONCLUDING REMARKS}

Are antibiotics naturally antibiotics? This question raised by Davies ${ }^{32}$ is related to the law underlying the constitution of a microbial community in a natural environment. This question can be interpreted as 'how diverse and complex are the modes of chemical communication among microorganisms?' The evidence has indicated that antibiotics exert a different biological action at a subinhibitory concentration. Hence, investigators should be more aware of the potential diversity inherent even in the known compounds with regard to their biological activity. Furthermore, the unknown compounds whose production does not take place under the conventional cultivation conditions harbor a lot of potential in terms of their structure and activity. Scientists in this field need to think about the real life of microorganisms in a natural environment in order to take full advantage of their abilities.

\section{CONFLICT OF INTEREST}

The authors declare no conflict of interest.

\section{ACKNOWLEDGEMENTS}

We thank Professor Morimasa Yagisawa for his editorial work on this special issue. In April 2000, we submitted a communication entitled 'Interspecific stimulatory events on antibiotic production and sporulation among Streptomyces species' to this journal. Then, the phrase 'Wide distribution of was added at the beginning of the title according to the comment of a reviewer. ${ }^{5}$ The suggestion was welcome, because it accurately reflected our observations. Now, we are pleased to dedicate this review to that originally anonymous reviewer, Professor Julian E Davies. His broad scope of expertise and deep insights have greatly advanced the field of applied microbiology. Julian is a man of big curiosity, and his personality always attracted scientists and students alike. We would like to celebrate his great career in science.

1 Katz, L. \& Baltz, R. H. Natural product discovery: past, present, and future. J. Ind. Microbiol. Biotechnol. 43, 155-176 (2016).

2 Hopwood, D. A. Therapeutic treasures from the deep. Nat. Chem. Biol. 3, 457-458 (2007)

3 Sonnenbichler, J., Dietrich, J. \& Peipp, H. Secondary fungal metabolites and their biological activities, V. Investigations concerning the induction of the biosynthesis of toxic secondary metabolites in basidiomycetes. Biol. Chem. Hoppe. Seyler. 375, 71-79 (1994).

4 Burgess, J. G., Jordan, E. M., Bregu, M., Mearns-Spragg, A. \& Boyd, K. G. Microbial antagonism: a neglected avenue of natural products research. J Biotechnol. 70, 27-32 (1999).

5 Ueda, $\mathrm{K}$ et al. Wide distribution of interspecific stimulatory events on antibiotic production and sporulation among Streptomyces species. J. Antibiot. 53, 979-982 (2000).

6 Horinouchi, S. \& Beppu, T. Hormonal control by A-factor of morphological development and secondary metabolism in Streptomyces. Proc. Jpn Acad. Ser. B Phys. Biol. Sci. 83, 277-295 (2007). 
7 Onaka, H., Mori, Y., Igarashi, Y. \& Furumai, T. Mycolic acid-containing bacteria induce natural-product biosynthesis in Streptomyces species. Appl. Environ. Microbiol. 77, 400-406 (2011).

8 Slattery, M., Rajbhandari, I. \& Wesson, K. Competition-mediated antibiotic induction in the marine bacterium Streptomyces tenjimariensis. Microb. Ecol. 41, 90-96 (2001).

9 Cueto, M. et al. Pestalone, a new antibiotic produced by a marine fungus in response to bacterial challenge. J. Nat. Prod. 64, 1444-1446 (2001).

10 Oh, D. C., Jensen, P. R., Kauffman, C. A. \& Fenical, W. Libertellenones A-D: induction of cytotoxic diterpenoid biosynthesis by marine microbial competition. Bioorg. Med. Chem. 13, 5267-5273 (2005).

11 Oh, D. C., Kauffman, C. A., Jensen, P. R. \& Fenical, W. Induced production of emericellamides $\mathrm{A}$ and $\mathrm{B}$ from the marine-derived fungus Emericella sp. in competing co-culture. J. Nat. Prod. 70, 515-520 (2007).

12 Meng, L. H. et al. Citrifelins A and B, citrinin adducts with a tetracyclic framework from cocultures of marine-derived isolates of Penicillium citrinum and Beauveria felina. J. Nat. Prod. 78, 2301-2305 (2015).

13 Degenkolb, T., Heinze, S., Schlegel, B., Strobel, G. \& Gräfe, U. Formation of new lipoaminopeptides, acremostatins $\mathrm{A}, \mathrm{B}$, and $\mathrm{C}$, by co-cultivation of Acremonium sp. Tbp-5 and Mycogone rosea DSM 12973. Biosci. Biotechnol. Biochem. 66 883-886 (2002).

14 Nonaka, K. et al. Enhancement of metabolites productivity of Penicillium pinophilum FKI-5653, by co-culture with Trichoderma harzianum FKI-5655. J. Antibiot. 64, 769-774 (2011).

15 Park, H. B., Kwon, H. C., Lee, C.H. \& Yang, H. O. Glionitrin A an antibiotic-antitumor metabolite derived from competitive interaction between abandoned mine microbes. J. Nat. Prod. 72, 248-252 (2009).

16 Zuck, K. M., Shipley, S. \& Newman, D. J. Induced production of $\mathrm{N}$-formyl alkaloids from Aspergillus fumigatus by co-culture with Streptomyces peucetius. J. Nat. Prod. 74, 1653-1657 (2011).

17 Whitt, J., Shipley, S. M., Newman, D. J. \& Zuck, K. M. Tetramic acid analogues produced by coculture of Saccharopolyspora erythraea with Fusarium pallidoroseum. J. Nat. Prod. 77, 173-177 (2014).

18 Hoshino, S. et al. Arcyriaflavin E, a new cytotoxic indolocarbazole alkaloid isolated by combined-culture of mycolic acid-containing bacteria and Streptomyces cinnamoneus NBRC 13823. J. Antibiot. 68, 342-344 (2015).
19 Hoshino, S. et al. Niizalactams A-C, multicyclic macrolactams isolated from combined culture of Streptomyces with mycolic acid-containing bacterium. J. Nat. Prod. 78, 3011-3017 (2015).

20 Hoshino, S., Wakimoto, T, Onaka, H. \& Abe, I. Chojalactones A-C, cytotoxic butanolides isolated from Streptomyces sp. cultivated with mycolic acid containing bacterium. Org. Lett. 17, 1501-1504 (2015).

21 Watsuji, T. O. et al. Identification of indole derivatives as self-growth inhibitors of Symbiobacterium thermophilum, a unique bacterium whose growth depends on coculture with a Bacillus sp. Appl. Environ. Microbiol. 73, 6159-6165 (2007).

22 Ueda, K. \& Beppu, T. Lessons from studies of Symbiobacterium thermophilum, a unique syntrophic bacterium. Biosci. Biotechnol. Biochem. 71, 1115-1121 (2007).

23 Ueda, K. \& Beppu, T. Syntrophic growth of Symbiobacterium in association with freeliving bacteria. In The Mechanistic Benefits of Microbial Symbionts. vol 2 (ed. Hurst, C. J.) (Springer, 2016).

24 Yamanaka, K. et al. Desferrioxamine E produced by Streptomyces griseus stimulates growth and development of Streptomyces tanashiensis. Microbiology. 151, 2899-2905 (2005).

25 Eto, D. et al. Divergent effects of desferrioxamine on bacterial growth and characteristics. J. Antibiot. 66, 199-203 (2013).

26 Traxler, M. F., Watrous, J. D., Alexandrov, T., Dorrestein, P. C. \& Kolter, R. Interspecies interactions stimulate diversification of the Streptomyces coelicolor secreted metabolome. MBio 4, e00459-e00513 (2013).

27 Amano, S. et al. Promomycin, a polyether promoting antibiotic production in Streptomyces spp. J. Antibiot. 63, 486-491 (2010).

28 Davies, J., Spiegelman, G. B. \& Yim, G. The world of subinhibitory antibiotic concentrations. Curr. Opin. Microbiol. 9, 445-453 (2006).

29 Amano, S.-I. et al. A cryptic antibiotic triggered by monensin. J. Antibiot. 64, 703 (2011).

30 Fujimoto, M., Chijiwa, M., Nishiyama, T., Takano, H. \& Ueda, K. Developmental defect of cytochrome oxidase mutants of Streptomyces coelicolor A3(2). Microbiology 162, 1446-1455 (2016)

31 Meng, L., Li, M., Yang, S. H., Kim, T. J. \& Suh, J. W. Intracellular ATP levels affect secondary metabolite production in Streptomyces spp. Biosci. Biotechnol. Biochem. 75, 1576-1581 (2011).

32 Davies, J. Are antibiotics naturally antibiotics? J. Ind. Microbiol. Biotechnol. 33, 496-499 (2006). 